\title{
Impactos na saúde da mulher mãe cuidadora de criança com câncer
}

\author{
Impacts on the health of caregiver mother of children with cancer \\ Impactos en la salud de la madre que cuida niño con cáncer
}

Recebido: 09/01/2021 | Revisado: 11/01/2021 | Aceito: 13/01/2021 | Publicado: 17/01/2021

\author{
Izabela da Silva Pinheiro \\ ORCID: https://orcid.org/0000-0003-1609-7778 \\ Universidade Federal do Estado do Rio de Janeiro, Brasil \\ E-mail: izabelapinheiro@live.com \\ Beatriz Cristina de Oliveira Guerra \\ ORCID: https://orcid.org/0000-0001-5992-5567 \\ Universidade Federal do Estado do Rio de Janeiro, Brasil \\ E-mail: beatrizcoliveira@edu.unirio.br \\ Leila Rangel da Silva \\ ORCID: https://orcid.org/0000-0003-1831-0982 \\ Universidade Federal do Estado do Rio de Janeiro, Brasil \\ E-mail: leila.silva@unirio.br \\ Patrícia Quintans Cundines Pacheco \\ ORCID: https://orcid.org/0000-0002-2256-3491 \\ Hospital Federal dos Servidores do Estado, Brasil \\ E-mail: patricia_quintans@ @otmail.com \\ Isabela da Costa Monnerat \\ ORCID: https://orcid.org/0000-0001-7658-8048 \\ Universidade Federal do Estado do Rio de Janeiro, Brasil \\ E-mail: belamonnerat@gmail.com \\ Selma Villas Boas Teixeira \\ ORCID: https://orcid.org/0000-0001-8799-0243 \\ Universidade Federal do Estado do Rio de Janeiro, Brasil \\ E-mail: selma.villasboas@globo.com \\ Eliza Cristina Macedo \\ ORCID: https://orcid.org/0000-0001-8824-9107 \\ Universidade Federal do Estado do Rio de Janeiro, Brasil \\ E-mail: macedo.unirio@gmail.com \\ Regina Claudia Veras \\ ORCID: https://orcid.org/0000-0003-4501-090X \\ Universidade Federal do Estado do Rio de Janeiro, Brasil \\ E-mail: regininhaveras@ hotmail.com
}

\begin{abstract}
Resumo
Objetivo: mapear as produções científicas quanto aos impactos para a saúde da mulher mãe cuidadora de criança/adolescente com câncer. Método: revisão integrativa da literatura, realizada nas bases informatizadas da Biblioteca Virtual de Saúde (BVS) em maio de 2020. A questão da pesquisa foi "Quais os impactos na saúde da mulher mãe cuidadora de criança/adolescente com câncer?". Resultados: Foram encontrados 46 artigos investigando a saúde da mulher-mãe cuidadora de criança com câncer e com a aplicação dos critérios de inclusão e exclusão foram selecionados 8 estudos. As mulheres-mães são as principais cuidadoras, o que acarreta na sobrecarga materna e no adoecimento expresso nas áreas: física, financeira, social, de informação, moral e emocional. Conclusão: A saúde da mãe-cuidadora é bastante afetada, sendo o adoecimento emocional o aspecto mais significativo. Essa é uma área de investigação ainda nova no cenário da enfermagem que precisa continuar avançando. Novas pesquisas precisam ser realizadas, em busca de um melhor entendimento acerca das necessidades dessa população.
\end{abstract}

Palavras-chave: Cuidadores; Mães; Neoplasias; Criança.

\begin{abstract}
Objective: map the scientific productions about the health impacts of women mother caregivers of children/adolescents with cancer. Methodology: integrative literature review, performed in the computerized bases of the Virtual Health Library (VHL) in May 2020. The research question was "What are the impacts on the health of women mother caregivers of children/adolescents with cancer? Results: 46 articles were found investigating the health of the motherchild caregiver with cancer and with the application of inclusion and exclusion criteria 8 studies were selected. The mother-women are the main caregivers, which causes the maternal overload and sickness expressed in the areas: physical, financial, social, information, moral and emotional. Conclusion: The health of the mother-caregiver is greatly affected, with emotional illness being the most significant aspect. This is an area of research that is still new in the
\end{abstract}


nursing scenario and needs to continue to advance. New research needs to be carried out, in search of a better understanding about the needs of this population.

Keywords: Caregivers; Mothers; Neoplasms; Child.

\begin{abstract}
Resumen
Objetivo: mapear las producciones científicas sobre los impactos en la salud de las mujeres madres cuidadoras de niños/adolescentes con cáncer. Metodología: revisión bibliográfica integradora, realizada en las bases de datos computarizadas de la Biblioteca Virtual de Salud (BVS) en mayo de 2020. La pregunta de la investigación fue "¿Cuáles son los impactos en la salud de las mujeres madres cuidadoras de niños/adolescentes con cáncer? Resultados: Se encontraron 46 artículos que investigaban la salud del cuidador madre-hijo con cáncer y con la aplicación de los criterios de inclusión y exclusión se seleccionaron 8 estudios. Las madres-mujeres son las principales cuidadoras, lo que da lugar a una sobrecarga materna y enfermedad que se expresa en los ámbitos: físico, financiero, social, informativo, moral y emocional. Conclusión: La salud de la madre-cuidadora se ve muy afectada, siendo la enfermedad emocional el aspecto más significativo. Se trata de un área de investigación que aún es nueva en el escenario de la enfermería y debe seguir avanzando. Es necesario realizar nuevas investigaciones, en busca de una mejor comprensión de las necesidades de esta población.
\end{abstract}

Palabras clave: Cuidadores; Madres; Neoplasias; Niño.

\title{
1. Introdução
}

Nos cenários de cuidado em instituições de saúde percebe-se as influências na saúde da mulher que cuida de um filho com câncer. A literatura aponta que a sobrecarga desta mulher é uma realidade. Os aspectos culturais impõem à mulher a responsabilidade, praticamente indelegável do cuidado ao filho doente (Pacheco, 2020).

Desde da época do Brasil colônia, as famílias são vistas pelo modelo patriarcal que têm o pai como figura de autoridade, e a mãe com o papel de educar os filhos e administrar a rotina doméstica e marido no âmbito privado. Com a revolução industrial e a entrada das mulheres no mercado de trabalho as atribuições aumentaram, uma vez que elas passaram a ocupar o espaço público assumindo uma profissão. Os anos se passaram e o olhar da sociedade continua sendo que a mulher que é mãe deverá ser a principal responsável, dedicada no cuidado e educação dos filhos, e com o rótulo que deverá ser uma boa esposa (Almeida, 2007). Afinal, será esse um dos motivos das mulheres serem apontadas como as principais cuidadoras do filho adoecido?

O câncer caracteriza-se por ser um conjunto de doenças que causa um crescimento desordenado de células e invadem tecidos e órgãos. Essas células são agressivas e podem espalhar-se (metástase) para outras regiões do corpo (Brasil, 2019). Estima-se que entre 2020 e 2022, surjam no Brasil 4.310 novos casos no sexo masculino e de 4.150 para o sexo feminino a cada ano (Brasil, 2020).

Considerando que o câncer é uma doença que demanda um cuidado diário e total dedicação do cuidador principal a criança doente, esta revisão integrativa tem como objetivo mapear as produções científicas quanto aos impactos na saúde da mulher mãe cuidadora de criança/adolescente com câncer.

\section{Metodologia}

Trata-se de uma revisão integrativa da literatura, que tem como propósito sintetizar os resultados da pesquisa procurando as semelhanças e diferenças entre os artigos para obter um aprofundamento no conhecimento do tema pesquisado baseando-se em estudos anteriores (de Souza, Marques-Vieira, Severino \& Antunes, 2017).

Esta revisão é composta por seis etapas: Estabelecimento da hipótese ou questão de pesquisa (etapa 1), Amostragem ou busca na literatura (etapa 2), Categorização dos estudos (etapa 3), Avaliação dos estudos incluídos na revisão (etapa 4), Interpretação dos resultados (etapa 5), Síntese do conhecimento ou apresentação da revisão (etapa 6) (Mendes, Silveira \& Galvão, 2008).

Na primeira etapa ficou estabelecida a seguinte questão de pesquisa: "Quais os impactos na saúde da mulher mãe cuidadora de criança/adolescente com câncer?”. Para a segunda fase da pesquisa, foi realizada a busca nas Bases de Dados 
informatizadas da Biblioteca Virtual de Saúde (BVS), com a utilização dos descritores de assunto contidos no Descritores em Ciências da Saúde (DeCS): "Cuidadores", "Mães", "Neoplasias" e "Criança" com o auxílio do operador booleano (AND).

O levantamento foi realizado em outubro de 2020. Optou-se por não estabelecer recorte temporal. Os critérios de inclusão foram artigos científicos originais nacionais e internacionais disponíveis na íntegra; publicados em português e inglês; independentemente do método de pesquisa e que respondessem a pergunta de pesquisa. Os critérios de exclusão foram artigos com cuidadores de crianças curadas ou com óbito por câncer; monografias; documentos de projetos; amostras duplicadas; artigos não disponíveis na íntegra; artigos com assunto principal não relacionado ao tema estudado.

Os artigos que apresentam no título outros cuidadores da criança com câncer, foram selecionados para a revisão por abordarem predominantemente a saúde da mulher- mãe.

\section{Resultados}

No cruzamento dos descritores foram encontrados 46 artigos investigando a saúde da mulher-mãe cuidadora de criança com câncer. Após o refinamento da busca baseada nos critérios de inclusão foram selecionadas 34 publicações destas foram excluídos 17 artigos por não disponibilizarem o texto completo, 07 artigos por não responderem a pergunta da pesquisa e 02 por serem duplicados. Dos artigos analisados, 08 compuseram o corpo desta revisão integrativa, como mostra o fluxograma a seguir (Figura 1).

Figura 1. Fluxograma da seleção dos estudos. Rio de Janeiro (RJ), Brasil, 2020.

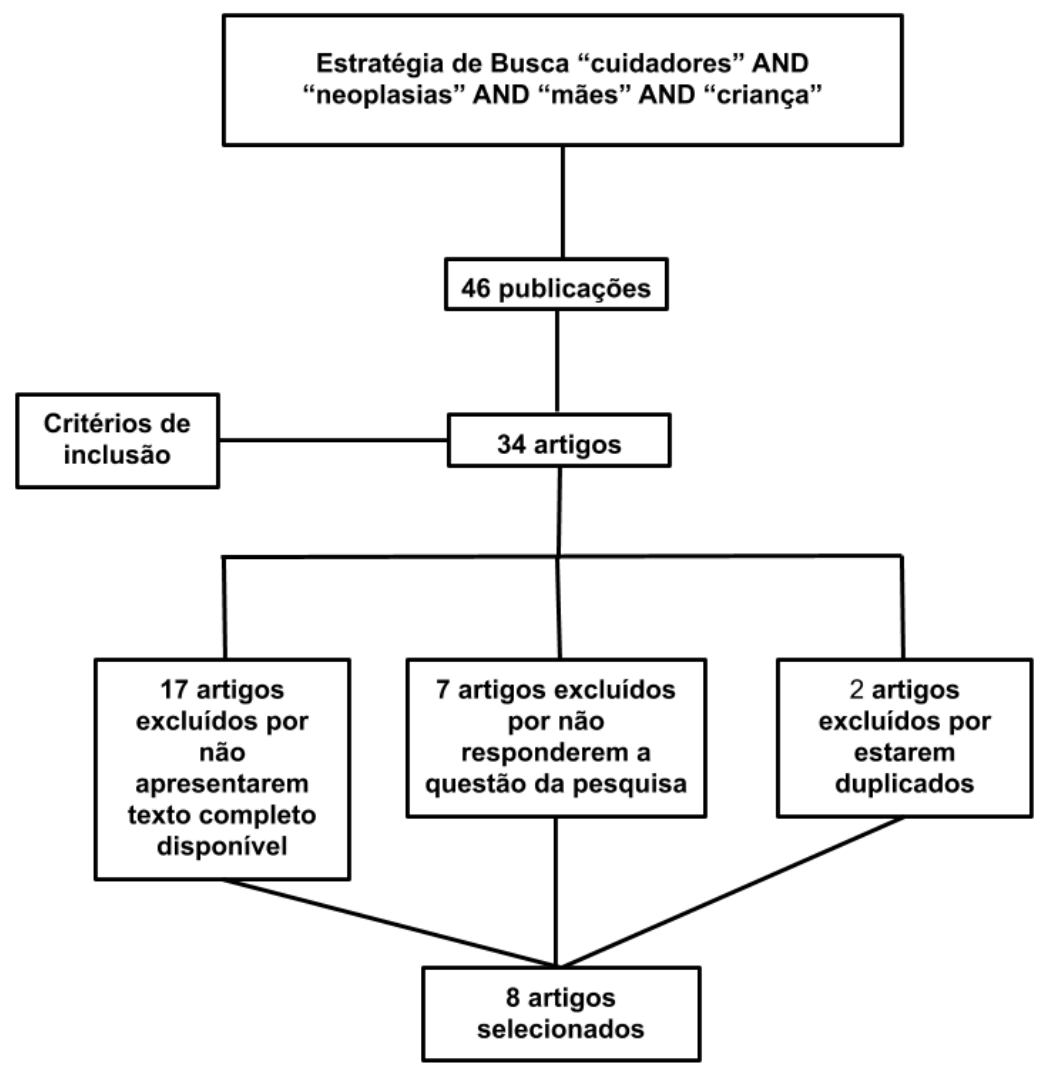

Fonte: Dados da pesquisa (2020). 
Os estudos encontrados nesta revisão integrativa foram classificados de acordo com o nível de evidência em conformidade com Oxford Centre Evidence-Based Medicine, que classifica as evidências de um estudo através de seu delineamento metodológico, como demonstrado na Tabela 1.

Tabela 1. Níveis de evidência científica segundo a Classificação de Oxford Centro de Medicina baseado em evidência (Março, 2009) versão traduzida. Rio de Janeiro (RJ), Brasil, 2020.

\begin{tabular}{|c|c|c|c|}
\hline \multicolumn{4}{|c|}{$\begin{array}{l}\text { Nivel de Evidência Científica por Tipo de Estudo - "Oxford Centre for } \\
\text { Evidence-based Medicine" - última atualização maio de } 2001\end{array}$} \\
\hline $\begin{array}{l}\text { Grau de } \\
\text { Recomendação }\end{array}$ & $\begin{array}{l}\text { Nivel de } \\
\text { Evidência }\end{array}$ & $\begin{array}{l}\text { Tratamento/ } \\
\text { Prevenção - Etiologia }\end{array}$ & Diagnóstico \\
\hline \multirow{3}{*}{ A } & $1 \mathrm{~A}$ & $\begin{array}{l}\text { Revisão Sistemática (com } \\
\text { homogeneidade) } \\
\text { de Ensaios Clinicos Controlados e } \\
\text { Randomizados }\end{array}$ & $\begin{array}{l}\text { Revisão Sistemática (com homogeneidade) } \\
\text { de Estudos Diagnósticos nivel } 1 \text { Critério } \\
\text { Diagnóstico de estudos nivel 1B, em } \\
\text { diferentes centros clinicos }\end{array}$ \\
\hline & $1 \mathrm{~B}$ & $\begin{array}{l}\text { Ensaio Clínico Controlado e } \\
\text { Randomizado com Intervalo de } \\
\text { Confiança Estreito }\end{array}$ & $\begin{array}{l}\text { Coorte validada, com bom padrão de } \\
\text { referência Critério Diagnóstico testado em um } \\
\text { único centro clínico }\end{array}$ \\
\hline & $1 \mathrm{C}$ & $\begin{array}{l}\text { Resultados Terapêuticos do tipo "tudo ou } \\
\text { nada" }\end{array}$ & $\begin{array}{l}\text { Sensibilidade e Especificidade próximas de } \\
100 \%\end{array}$ \\
\hline \multirow{5}{*}{ B } & $2 \mathrm{~A}$ & $\begin{array}{l}\text { Revisão Sistemática (com } \\
\text { homogeneidade) } \\
\text { de Estudos de Coorte }\end{array}$ & $\begin{array}{l}\text { Revisão Sistemática (com homogeneidade) } \\
\text { de estudos diagnósticos de nivel > } 2\end{array}$ \\
\hline & 2B & $\begin{array}{l}\text { Estudo de Coorte (incluindo Ensaio } \\
\text { Clinico } \\
\text { Randomizado de Menor Qualidade) }\end{array}$ & $\begin{array}{l}\text { Coorte Exploratória com bom padrāo de } \\
\text { Referência Critério Diagnóstico derivado ou } \\
\text { validado em amostras fragmentadas } \\
\text { ou banco de dados }\end{array}$ \\
\hline & $2 \mathrm{C}$ & $\begin{array}{l}\text { Observação de Resultados Terapêuticos } \\
\text { (outcomes research) } \\
\text { Estudo Ecológico }\end{array}$ & \\
\hline & $3 A$ & $\begin{array}{l}\text { Revisão Sistemática (com } \\
\text { homogeneidade) } \\
\text { de Estudos Caso-Controle }\end{array}$ & $\begin{array}{l}\text { Revisão Sistemática (com homogeneidade) } \\
\text { de estudos diagnósticos de nivel > 3B }\end{array}$ \\
\hline & 3B & Estudo Caso-Controle & $\begin{array}{l}\text { Seleção não consecutiva de casos, ou } \\
\text { padrão de referência aplicado de forma } \\
\text { pouco consistente }\end{array}$ \\
\hline C & 4 & $\begin{array}{l}\text { Relato de Casos (incluindo Coorte ou } \\
\text { Caso-Controle de menor qualidade) }\end{array}$ & $\begin{array}{l}\text { Estudo caso-controle; ou padrão de referência } \\
\text { pobre ou não independente }\end{array}$ \\
\hline D & 5 & $\begin{array}{r}\text { Opiniāo desprovida de avaliação critica ou } \\
\text { ou estuc }\end{array}$ & $\begin{array}{l}\text { aseada em matérias básicas (estudo fisiológico } \\
\text { com animais) }\end{array}$ \\
\hline
\end{tabular}

Fonte: Oxford Centre for Evidence-based Medicine - Levels of Evidence (March 2009).

No quadro a seguir é possível observar a distribuição dos artigos utilizados levando em consideração o ano de publicação, nível de evidência, periódico em que foi publicado e os objetivos dos estudos. 
Quadro 1. Distribuição dos estudos selecionados. Rio de Janeiro (RJ), Brasil, 2020.

\begin{tabular}{|c|c|c|c|c|c|}
\hline $\begin{array}{l}\text { IDENTIFICA } \\
\text { ÇÃO DO } \\
\text { ARTIGO }\end{array}$ & TÍTULO & NO & $\begin{array}{l}\text { NÍVEL } \\
\text { DE } \\
\text { EVIDÊN } \\
\text { CIA }\end{array}$ & PERIÓDICO & OBJETIVOS \\
\hline A1 & $\begin{array}{l}\text { Meu papel e } \\
\text { responsabilidade: as } \\
\text { perspectivas das mães } \\
\text { sobrecarregadas com o } \\
\text { cuidado dos filhos com } \\
\text { câncer }\end{array}$ & 020 & 4 & $\begin{array}{l}\text { Revista da Escola } \\
\text { de Enfermagem } \\
\text { da USP. }\end{array}$ & $\begin{array}{l}\text { Compreender a experiência da } \\
\text { sobrecarga materna no cuidado } \\
\text { dos filhos com câncer do ponto } \\
\text { de vista da mãe }\end{array}$ \\
\hline A2 & $\begin{array}{l}\text { Vulnerabilidade ao } \\
\text { estresse: pais cuidadores } \\
\text { de filhos com câncer }\end{array}$ & 019 & 4 & $\begin{array}{l}\text { Revista de } \\
\text { Pesquisa Cuidado } \\
\text { é Fundamental } \\
\text { Online }\end{array}$ & $\begin{array}{l}\text { Investigar o estresse vivenciado } \\
\text { por pais ou mães que cuidam de } \\
\text { filhos com câncer }\end{array}$ \\
\hline A3 & $\begin{array}{l}\text { Perfil nutricional e } \\
\text { qualidade de vida de } \\
\text { cuidadores de crianças e } \\
\text { adolescentes com câncer }\end{array}$ & 018 & $2 \mathrm{~B}$ & $\begin{array}{l}\text { Clinical \& } \\
\text { Biomedical } \\
\text { Research }\end{array}$ & $\begin{array}{l}\text { Verificar o estado nutricional, o } \\
\text { consumo alimentar de energia e } \\
\text { macronutrientes e a QV de } \\
\text { cuidadores de crianças e } \\
\text { adolescentes com câncer nos } 6 \\
\text { primeiros meses de tratamento } \\
\text { oncológico }\end{array}$ \\
\hline A4 & $\begin{array}{l}\text { Desvelando a } \\
\text { experiência de mães de } \\
\text { crianças com câncer }\end{array}$ & 016 & 4 & $\begin{array}{l}\text { Revista de } \\
\text { Enfermagem do } \\
\text { Centro Oeste } \\
\text { Mineiro }\end{array}$ & $\begin{array}{l}\text { Compreender o cotidiano de } \\
\text { mães cuidadoras de crianças com } \\
\text { câncer }\end{array}$ \\
\hline A5 & $\begin{array}{l}\text { Qualidade de Vida e } \\
\text { Sobrecarga de } \\
\text { Cuidadores de Crianças } \\
\text { com Câncer }\end{array}$ & 014 & 4 & $\begin{array}{l}\text { Psicologia: } \\
\text { Ciência e } \\
\text { Profissão }\end{array}$ & $\begin{array}{l}\text { Verificar a relação entre a } \\
\text { qualidade de vida e a sobrecarga } \\
\text { em cuidadores de crianças } \\
\text { portadoras de neoplasia e } \\
\text { assistidas em uma casa de apoio }\end{array}$ \\
\hline A6 & $\begin{array}{l}\text { Sobrecarga e qualidade } \\
\text { de vida de cuidadores de } \\
\text { criança e adolescentes } \\
\text { com câncer em }\end{array}$ & 012 & 4 & $\begin{array}{l}\text { Acta Paulista de } \\
\text { Enfermagem }\end{array}$ & $\begin{array}{l}\text { Avaliar a sobrecarga de cuidado } \\
\text { e a qualidade de vida (QV) de } \\
\text { cuidadores de } \\
\text { crianças/adolescentes com câncer } \\
\text { durante tratamento }\end{array}$ \\
\hline
\end{tabular}




\begin{tabular}{|c|c|c|c|c|c|}
\hline & $\begin{array}{l}\text { tratamento } \\
\text { quimioterápico }\end{array}$ & & & & $\begin{array}{l}\text { quimioterápico e relacioná-las } \\
\text { entre si e aos dados } \\
\text { sociodemográficos }\end{array}$ \\
\hline A7 & $\begin{array}{l}\text { Lutos e lutas: } \\
\text { reestruturações familiares } \\
\text { diante do câncer em uma } \\
\text { criança/adolescente }\end{array}$ & 011 & 4 & $\begin{array}{l}\text { Psicologia } \\
\text { Argumento }\end{array}$ & $\begin{array}{l}\text { Compreender qual é a } \\
\text { significação que os } \\
\text { pais/cuidadores de crianças } \\
\text { diagnosticadas com câncer } \\
\text { atribuem às relações entre esse } \\
\text { diagnóstico e a sua dinâmica } \\
\text { familiar }\end{array}$ \\
\hline A8 & $\begin{array}{l}\text { Parental stress when } \\
\text { caring for a child with } \\
\text { cancer in Jordan: a cross- } \\
\text { sectional survey. }\end{array}$ & 012 & $2 \mathrm{C}$ & $\begin{array}{l}\text { Health and } \\
\text { Quality of Life } \\
\text { Outcomes }\end{array}$ & $\begin{array}{l}\text { Explorar os níveis de estresse de } \\
\text { pais jordanianos cuidando de } \\
\text { uma criança com câncer, a fim de } \\
\text { identificar as necessidades } \\
\text { psicológicas de pais neste } \\
\text { ambiente e explorar como os } \\
\text { níveis de estresse de mães e pais } \\
\text { podem diferir. }\end{array}$ \\
\hline
\end{tabular}

Fonte: Dados da pesquisa (2020).

Os oito (100\%) artigos encontrados foram publicados entre os anos de 2011 a 2020, ressaltando que quatro (25\%) periódicos que publicaram artigos associados à saúde da mulher-mãe de criança com câncer são da área de enfermagem, segundo o Quadro 1.

Com relação à procedência da produção, observa-se a distribuição das pesquisas acerca da saúde da mulher-mãe de criança com câncer, conforme Tabela 2.

Tabela 2. Distribuição das publicações sobre saúde da mulher-mãe cuidadora de criança com câncer por área de procedência. Rio de Janeiro (RJ), Brasil, 2020.

\begin{tabular}{ccc}
\hline Procedência & Frequência & $\%$ \\
\hline Brasil - Paraíba & 3 & 37,5 \\
Brasil - Rio Grande do Sul & 2 & 25 \\
Brasil - São Paulo & 2 & 25 \\
Jordânia & 1 & 12,5 \\
Total & 8 & 100 \\
\hline
\end{tabular}

Fonte: Autores (2020). 
Destaca-se que a maioria dos estudos - sete $(87,5 \%)$ - procede do Brasil, tendo apenas uma publicação internacional, na Jordânia $(12,5 \%)$ sobre a temática.

A distribuição de publicações sobre saúde da mulher mãe-cuidadora de criança com câncer por área do conhecimento é apresentada na Tabela 3. A maioria das pesquisas revisadas procede da enfermagem sendo representada em cinco $(62,5 \%)$ publicações.

Tabela 3. Distribuição da quantidade de publicações sobre saúde da mulher mãe-cuidadora de criança com câncer por área do conhecimento. Rio de Janeiro (RJ), Brasil, 2020.

\begin{tabular}{ccc}
\hline Procedência & Frequência & $\%$ \\
\hline Enfermagem & 5 & 62,5 \\
Psicologia & 2 & 25 \\
Medicina & 1 & 12,5 \\
Total & 8 & 100 \\
\hline
\end{tabular}

Fonte: Autores (2020).

Nos oito (100\%) artigos, os dados com relação a saúde da mulher mãe cuidadora foram coletados a partir de entrevistas ou questionários, o que é valioso para a pesquisa, pois assim os resultados possuem as singularidades e percepções de cada uma das mulheres.

\section{Discussão}

Os estudos mostraram os desafios que a mulher-mãe cuidadora do filho com câncer enfrenta. Foi observado que a maioria é sobrecarregada por serem as únicas responsáveis pela criança ou adolescente adoecido, mulheres que deixaram sua vida pessoal de lado para se dedicar exclusivamente ao filho.

Quanto aos participantes dos estudos a maioria era da idade média de 35 anos do sexo feminino, e muitas das vezes desempregadas por dedicarem seu tempo ao filho adoecido. Andrade, Alves, Melo e Rodrigues (2014) mostra em seu estudo que faltar ao trabalho e deixar de trabalhar foram as queixas mais recorrentes.

Ser mãe de uma criança com câncer ultrapassa as dimensões do cuidado, expressando-se por comportamentos e ações mediadas pelo tempo e pela necessidade de afastar a possibilidade de perder seu filho. Logo, a situação remete que a mãe supere os desafios e dificuldades que são colocados pelo estado de saúde da criança e necessita ser compreendida nas diversas fases evolutivas da doença (Costa et al, 2016).

A maior parte dos estudos mostra que o adoecimento e a hospitalização de um filho mudam o cotidiano familiar, além da vida pessoal. A mãe cuidadora vivencia diversas situações, como escassez de recursos financeiros, exercício de múltiplas funções na família, sentimentos de desamparo, perda de controle, exclusão e sobrecarga (Costa et al, 2016).

Com toda a mudança do cotidiano familiar os laços afetivos entre os filhos sadios e até mesmo entre o casal são abalados. Essa divisão da família, devido às exigências impostas pelo tratamento, pode promover uma sensação de perda da filiação, uma vez que os pais tendem a sentirem-se responsáveis pela coesão familiar. Tal condição gera a sensação de impotência e o 
sentimento de que os cuidadores estão sendo negligentes e irresponsáveis (Quintana, Wottrich, Camargo, de Quadros Cherer e Ries, 2011).

Os oito trabalhos selecionados apontam o fato da mulher-mãe cuidadora muitas vezes não tem o apoio do cônjuge ou de outros familiares, o que leva a uma enorme sobrecarga. Rubira, Marcon, Belasco, Gaíva e Espinosa (2012) em seu estudo realizou uma análise multivariada dos dados que permitiu identificar aspectos psicossociais e físicos (estado geral de saúde, aspectos sociais, vitalidade, problemas de saúde e custos extras em razão da função de cuidador), preditores independentes da sobrecarga percebida pelos cuidadores e que responderam a 36,0\% dessa sobrecarga.

Os estudos selecionados nesta revisão se apresentam em pesquisas quantitativas e qualitativas, tendo como autores predominantemente enfermeiros e psicólogos. Na prática esses profissionais são os que mais participam do dia a dia dessa mulher-mãe cuidadora, assim reconhecem a necessidade de discutir cada vez mais sobre a saúde dessas mães, uma vez que muitas vezes elas à negligenciam pois todo o seu tempo e esforços são dedicados à vida do filho com câncer.

Quanto ao perfil das mães cuidadoras do filho com câncer, a idade média é em torno dos 35 anos. Em relação ao estado civil em sua maioria são casadas, no entanto, segundo o estudo de Zortéa, Lazeri, Behling, Cruz e Gregianin (2018), após 6 meses do diagnóstico de câncer do filho, a porcentagem de divórcios duplicou, tal fato pode ser atribuído ao distanciamento do casal no processo de doença.

A respeito da ocupação das participantes dos estudos, a maioria informa pedir demissão ou parar seus estudos, estão desempregadas, dedicando seu tempo exclusivamente ao tratamento do filho.

A escolaridade dessas mulheres-mães é majoritariamente o ensino fundamental completo, contudo o número de mães que não completaram o ensino fundamental e até mesmo que não foram alfabetizadas é bem alto. Isso refletiu em uma sensação de impotência de muitas mulheres, que se viam perdidas e desamparadas no hospital por não entenderem a linguagem técnica que os profissionais frequentemente utilizavam, fenômeno que Vieira e Cunha (2020) chamou de "information overload", que acontece quando há um excesso de informações recebidas ao mesmo tempo e de forma inadequada, o que gera confusão nessas mulheres-mães sendo uma fonte de tensão para elas.

\section{Saúde física da mulher-mãe cuidadora}

Vieira e Cunha (2020) apontam que cuidadores de criança com câncer sofrem mais estressores e sobrecarga do que os de crianças saudáveis. Quando o cuidador é um familiar, como as mães, esse estresse fica mais evidenciado, uma vez que a expectativa ao cuidar é maior, assim como a auto cobrança para fazer sempre mais (Andrade et al., 2014).

A sobrecarga física da mulher-mãe cuidadora é definida pela fadiga decorrente do acúmulo de tarefas que envolvem o cuidado próprio, as funções que ela têm com os outros membros da família, as atividades domésticas e com as dificuldades diretamente e indiretamente decorrentes da doença do filho, como os cuidados que ele demanda durante uma internação, a locomoção até as sessões de quimioterapia e radioterapia e as atividades de vida diária como banho, alimentação, etc (Vieira e Cunha, 2020; Andrade et al., 2014; Zortéa et al, 2018).

Foi observado que meses após o diagnóstico de câncer na criança, o Índice de Massa Corporal (IMC) da mulher-mãe cuidadora sofre elevações consideráveis, como um aumento de mais de 30\% no número de obesos em apenas 6 meses. O risco de doença cardiovascular não apresentou aumento significativo, porém a prática de atividades físicas diminuiu pela metade (Zortéa et al, 2018).

Apesar de ser reconhecido, esse estresse materno muitas vezes não é discutido, pois para as mães cuidarem do filho doente é gratificante, mesmo que seja extremamente difícil aceitar que a condição da criança ou adolescente com câncer afeta negativamente na sua saúde (Andrade et al., 2014). Outros sintomas físicos podem surgir decorrentes desse estresse, como hipertensão, problemas infecciosos, indigestão, úlceras, cefaleia e insônia, e uma forma de amenizar esses efeitos é através de 
massagens antiestresse (Fontes et al., 2019).

Diante dessas circunstâncias que a mulher-mãe cuidadora enfrenta, é necessária uma equipe multiprofissional que facilite o acesso dela às práticas de saúde com acolhimento e escuta ativa, desenvolvendo ou a encaminhando para atividades que auxiliem no cuidado e promova um momento de relaxamento como o uso das Práticas Integrativas e Complementares (Brasil, 2006).

\section{Saúde emocional da mulher-mãe cuidadora}

Foi identificado que 100\% das mulheres-mães cuidadoras de crianças em tratamento do câncer são vulneráveis ao estresse devido ao desgaste emocional que a doença causa, o que requer um acompanhamento psicológico para auxiliar os sentimentos do paciente, familiares e até mesmo dos profissionais envolvidos com o tratamento (Fontes et al., 2019). Os pais das crianças também apresentaram níveis de estresse, mas eles eram significativamente mais baixos do que os das mulheres (Masa'Deh, Collier \& Hall, 2012).

A internação do filho também gera uma sensação de aprisionamento e impotência, pois precisam seguir as regras do espaço hospitalar, e não tem assim a liberdade e bem- estar que a casa pode promover (Quintana el at., 2011). As inúmeras informações que essa mulher recebe durante o diagnóstico e tratamento do filho são por si só outro fator que abala o emocional delas, uma vez que geram angústia e medo, e quando ela é passada de forma acumulada e em uma linguagem excludente com quem é leigo, funciona como mais uma fonte de tensão no meio de tantos problemas.

A mulher-mãe cuidadora relata uma sobrecarga moral gerada pelo preconceito que os filhos com câncer sofrem tanto dos profissionais como da população em geral que erroneamente muitas vezes tem medo de se contaminarem com a doença. Esse peso moral também fica evidenciado através da cobrança pessoal e dos familiares para serem mães exímias (Vieira e Cunha, 2020).

Isso tudo só gera mais estresse e ansiedade nessas mulheres tão carregadas emocionalmente, que vivenciam uma dualidade de sentimentos, onde se sentem esgotadas devido a toda responsabilidade que carregam e por outro lado apresentam satisfação na tarefa de ser a pessoa responsável para cuidar do filho. Com isso, a fé é muitas vezes a única saída que elas enxergam, atribuindo a Deus a força para continuar os cuidados (Andrade, 2014).

De acordo com Macedo, Silva, Paiva e Ramos (2015), é necessário incentivar os pontos fortes das mulheres-mães cuidadoras, bem como, promover grupos de encontro entre elas. Além disso, enfatizar o senso de empoderamento delas com o objetivo de aliviar sentimentos de autoeficácia prejudicada e consequente sobrecarga. Contudo, é preciso também perceber a satisfação das mães cuidadoras com o tratamento disponibilizado e também as dificuldades por elas enfrentadas para acessar os serviços especializados e redes de apoio.

Com base no exposto, a mulher-mãe cuidadora de criança/adolescente com câncer necessita de um cuidado multiprofissional com o objetivo de acolher suas dores, angústias e medos; exercer uma escuta ativa estabelecendo um vínculo de confiança.

\section{Relacionamento interpessoal da mulher-mãe cuidadora}

Houve quase que unanimidade nos estudos ao mostrar que a falta de envolvimento familiar e a falta de apoio do companheiro interferem negativamente na saúde do cuidador.

Os estudos mostraram que a maioria das cuidadoras eram mulheres, assim, evidencia-se o papel atribuído à mulher culturalmente em que o cuidado é visto muitas vezes como uma extensão das atividades domésticas enquanto aos pais, a figura coadjuvante, cabia prover recursos financeiros para a família (Andrade, 2014).

De acordo com Quintana et al (2011), esse papel dos pais é compreendido por alguns como necessário e por isso 
valorizado pelo cumprimento de suas atribuições. Contudo, a cuidadora ainda permanece com o sentimento de abandono e desamparo. No estudo de Costa et al (2016) as participantes externaram sobre o sentimento de privação sexual devido ao distanciamento do casal, assim, aumentando o estresse conjugal.

A respeito da relação com seus filhos sadios, a mãe-cuidadora se culpabiliza pelos efeitos que geram nos outros filhos. Costa et al (2016) mostra que há evidências de desprezo e ciúmes por parte dos filhos sem diagnóstico, provocados pelo cuidado excessivo do irmão com câncer. Tal situação gera sensação de impotência e irresponsabilidade nas mães.

O estudo de Quintana et al (2011) destacou que o papel de outros familiares se resume ao suporte afetivo e aos afazeres domésticos. Visto que as mães não se sentem confortáveis ao deixar outras pessoas cuidarem de seu filho, como mostra o estudo de Fontes et al (2019).

Assim, o papel de multitarefas da mulher-mãe gera efeitos negativos fisicamente e psicologicamente, e elas acabam depositando todas as suas esperanças nos profissionais da saúde que acompanham sua luta diariamente. Além disso, todos esses fatores geram estresse no ambiente familiar da criança/adolescente com câncer (Fontes et al, 2001).

A vida social de toda a família fica abalada, uma vez que, passeios, festas ou viagens se tornam eventos raros devido à baixa imunidade da criança com câncer. (Andrade et al., 2014) Todavia, as relações da mulher-mãe cuidadora são as que mais sofrem mudanças, pois ela fica a maior parte do tempo com o filho, e em alguns casos o acesso às redes sociais é a única forma que elas encontram para manter uma relação social (Vieira \& Cunha, 2020).

Outro aspecto que fica prejudicado na vida dessa mulher é o financeiro, afinal, ela precisa alterar seu horário de trabalho, afastar-se dele por um tempo ou até mesmo pedir demissão, o que acaba gerando uma dependência financeira, seja do marido ou de outro membro da família (Andrade et al., 2014).

Além dos profissionais pertencentes a diversas áreas do conhecimento, é fundamental no cuidado à essa mulher, o envolvimento de uma rede de apoio com parentes, vizinhos, amigos e até mesmo instituições e grupos de ajuda visando uma troca de experiências, compartilhamento de informações e materiais de apoio e também como meio de promoção à saúde emocional dessa mãe.

\section{Limitação do estudo}

A limitação desta investigação é a escassez de publicações nacionais e internacionais que abordem a saúde da mãe que cuida da criança/adolescente com câncer. Faz-se necessário que outros estudos sejam realizados para que se possa discutir e propor mudanças no cuidado à saúde da mulher- mãe cuidadora.

\section{Contribuições do estudo para a prática}

Este estudo pode auxiliar no desenvolvimento e implementação de estratégias efetivas, que aliviem a sobrecarga da mulher-mãe cuidadora, e também para que tenhamos um cuidado multidisciplinar voltado para essa clientela. Assim, reforça-se a importância de reuniões de implementação de uma assistência/cuidado à saúde da mulher baseada em evidências científicas.

\section{Conclusão}

Esta revisão integrativa que trata dos impactos na saúde da mulher mãe cuidadora de criança com câncer, oportunizou as pesquisadoras a conhecerem os principais achados na literatura científica sobre a temática e a necessidade de preenchimento nas lacunas do conhecimento apresentadas como por exemplo de pensar nas práticas integrativas e complementares, nos serviços de atenção terciária para melhoria da saúde dessas mães.

Apesar dos agravos à saúde física serem apontados, a maioria dos estudos estão limitados aos aspectos de ordem emocional. Ressalta-se a importância do preparo de profissionais de saúde, sensibilizando-os, para que esses sejam capazes de 
amparar essas mães, fortalecendo vínculo com a escuta ativa e o acolhimento. Uma das formas seria incentivando e promovendo grupos de apoio, visto que o contato com outras mães cuidadoras minimiza o sentimento de solidão e desamparo ao longo da hospitalização do filho.

A questão financeira na vida dessas mulheres é algo que precisa de atenção. As dificuldades materiais são uma realidade, dado que a dependência do cônjuge e/ou outros familiares que essas mulheres acabam tendo, pode gerar sentimento de impotência, o que influi diretamente no emocional que pode causar consequências na sua saúde física.

Portanto, um fator prejudicado por exemplo estresse, serve de gatilho para o detrimento de outros comprometimentos físicos, mentais e espirituais, transformando a vida dessa mulher-mãe cuidadora em um ciclo de extrema sobrecarga que pode se estender para além da cura ou morte do filho.

Sugere-se que novas pesquisas precisam ser realizadas, em busca de um melhor entendimento acerca das necessidades dessa população, principalmente, no que diz respeito às relações dela enquanto mulher, suas metas profissionais, convivência social, vida sexual e desejos que não tem relação com a doença do filho, além das dificuldades enfrentadas por ela para o acesso a uma rede de suporte eficaz. A saúde e a sobrecarga da mãe-cuidadora, são uma área de investigação, no cenário da enfermagem, que precisa continuar avançando.

\section{Agradecimentos}

Os autores agradecem a Universidade Federal do Estado do Rio de Janeiro (UNIRIO) e ao Conselho Nacional de Desenvolvimento Científico e Tecnológico (CNPq), pelo Programa Institucional de Bolsas de Iniciação Científica (PIBIC) que possibilitou a execução desse estudo.

\section{Referências}

Almeida, L. S. D. (2007). Mãe, cuidadora e trabalhadora: as múltiplas identidades de mães que trabalham. Revista do Departamento de Psicologia. UFF, 19(2), 411-422.

Andrade, S. F. D. O., Alves, R. F., Melo, M. D. O., \& Rodrigues, M. J. (2014). Qualidade de vida e sobrecarga de cuidadores de crianças com câncer. Psicologia: ciência e profissão, 34(4), 1014-1031.

Brasil. (2006). Ministério da Saúde. Portaria no 971, de 03 de Maio de 2006. https://bvsms.saude.gov.br/bvs/saudelegis/gm/2006/prt0971_03_05_2006.html

Brasil (2019). O que é o câncer? 2019. https://www.inca.gov.br/o-que-e-cancer

Brasil (2020). Instituto Nacional do Câncer. Estimativa 2020-Síntese de Resultados e Comentários. https://www.inca.gov.br/estimativa/sintese-de-resultadose-comentarios

Centre for Evidence-based Medicine (2009). Oxford Center for Evidence-Based Medicine: Levels of Evidence (março de 2009). https://www.cebm.ox.ac.uk/resources/levels-of-evidence/oxford-centre-for-evidence-based-medicine-levels-of-evidence-march-2009

Costa, M. A. D. J. D., Agra, G., Souza Neto, V. L. D., Silva, B. C. O. D., Braz, L. C. D. S. B., \& Mendonça, A. E. O. D. (2016). Desvelando a experiência de mães de crianças com câncer. Rev. enferm. Cent.-Oeste Min, 2052-2065.

Fontes, A. L. C., Patrício, A. C. F. D. A., Lima, L. M. D., Santos, T. D. D., Nascimento, L. B. M. D., \& Silva, R. A. R. D. (2019). Vulnerabilidade ao estresse: pais cuidadores de filhos com câncer. Rev. pesqui. cuid. fundam.(Online), 857-861.

Macedo, E. C., Silva, L. R. D., Paiva, M. S., \& Ramos, M. N. P. (2015). Sobrecarga e qualidade de vida de mães de crianças e adolescentes com doença crônica: revisão integrativa. Revista Latino-Americana de Enfermagem, 23(4), 769-777.

Masa'Deh, R., Collier, J., \& Hall, C. (2012). Parental stress when caring for a child with cancer in Jordan: a cross-sectional survey. Health and Quality of Life Outcomes, $10(1), 88$.

Mendes, K. D. S., Silveira, R. C. D. C. P., \& Galvão, C. M. (2008). Revisão integrativa: método de pesquisa para a incorporação de evidências na saúde e na enfermagem. Texto \& contexto enfermagem, 17(4), 758-764.

Pacheco, P. Q. C. A mulher-mãe cuidadora de criança e adolescente com câncer: abordagem teórica de Levine. Tese (Doutorado) - Universidade Federal do Estado do Rio de Janeiro, Programa de Pós-Graduação em Enfermagem e Biociências. Rio de Janeiro, 2020. $244 \mathrm{f}$.

Quintana, A. M., Wottrich, S. H., Camargo, V. P., de Quadros Cherer, E., \& Ries, P. K. (2011). Lutos e lutas: Reestruturações familiares diante do câncer em uma criança/adolescente. Psicologia Argumento, 29(65). 
Research, Society and Development, v. 10, n. 1, e31510111828, 2021

(CC BY 4.0) | ISSN 2525-3409 | DOI: http://dx.doi.org/10.33448/rsd-v10i1.11828

Rubira, E. A., Marcon, S. R., Belasco, A. G. S., Gaíva, M. A. M., \& Espinosa, M. M. (2012). Sobrecarga e qualidade de vida de cuidadores de criança e adolescentes com câncer em tratamento quimioterápico. Acta Paul Enferm., 25(4), 567-573.

de Sousa, L. M. M., Marques-Vieira, C. M. A., Severino, S. S. P., \& Antunes, A. V. (2017). A metodologia de revisão integrativa da literatura em enfermagem. No21 Série 2-Novembro 2017, 17.

Vieira, A. C., \& Cunha, M. L. D. R. (2020). My role and responsibility: mothers' perspectives on overload in caring for children with cancer. Revista da Escola de Enfermagem da USP, 54.

Zortéa, J., Lazeri, L. L., Behling, E. B., Cruz, L. B. D., \& Gregianin, L. J. (2018). Perfil nutricional e qualidade de vida de cuidadores de crianças e adolescentes com câncer. Clinical and biomedical research. Porto Alegre. 38(1) 74-80. 